Casa Nacional del Niōn

Cáted ta Extraordinatia da Pediatria.

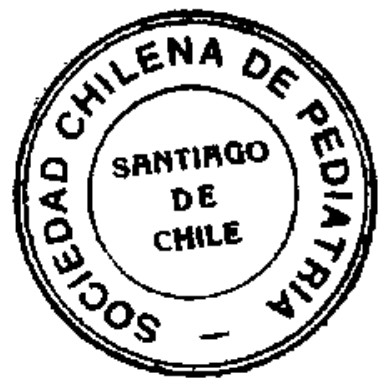

Prof. Dr. Ariztía.

\title{
alimentacion artificial en el lactante en el PRIMER TRIMESTRE
}

Por el Dr. GONZALO MORAGA F.

.Tefe de Servicio.

Razones múltipiles obligan al empleo en el lactante en ol primer trimestre de la vida, de la alimentación artificial. Su perfeccionamiento ha reducido al mínimo sus peligros ditec. tos e indirectos. Eso no quiete decir que en ningún caso sea superior a la alimentación natural materna.

La alimentación artificial en el lactante, en cualquier período de su vida y con mayor razón en el primer trimestre. requiere observación minuciosa tanto en el niño mismo en cuanto se refiere a su crecimiento ponderal, estatural, psiqui-

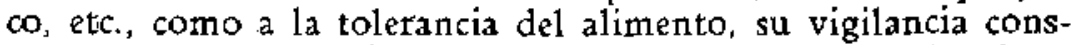
tante en su preparación y componente, ya que en el primer trimestre de la vida las defensas orgánicas son mínimas. y los fracasos no se deben a su calidad o cantidad, sino a sus componentes, pues no basta que se produzca aumento en el peso y talla, de acuerdo a su edad, sino que principalmente satisfaga los requerimientos cuantitativos y cualitativos del organismo los que traen consigo una mejor inmunidad y prevención de enfermedades propias de esta edad: raquitismo, anemia y cuadros carenciales diversos que se deben, en la gran mayoría de los casos, no a falta de vitaminas, sino a deficiencia de algunos de los componentes básicos en la composición quimica del alimento, sea albúmina, grasa o hidratos de carbono que deben contener.

Si estudiamos la leche de mujer y de vaca. vemos quz sus componentes, término medio y en números redondos son: albúmina: $1 \%$ en la leche de mujer, $3 \%$ en la de vaca; 
H. de C.: $7 \%$ en la de mujer, $3 \%$ en la de vaca; grasas: $3.5 \%$ en ambas. Durante mucho tiempo se creyó que la mayor proporción de albúmina en la leche de vaca era causa de su difícil digestibilidad y para corregir sus efectos desfavorables se usaba en diluciones que hacian disminuir sus com. ponentes en $\mathrm{H}$. de C. y grasas, produciendo la carencia de los materiales histoplásticos más importanites. Hoy día se ha reaccionado usando mezclas alimenticias a base de leche de vaca, considerando las proporciones necesarias de sus componentes principales, pues cada uno de ellos llena una función físico-química y biológica en el organismo del lactante. Asi, por ejemplo, si damos un alimento pobre en grasa y rico en $\mathrm{H}$. de C. se obtiene aumento de peso satisfactorio, pero pronto vemos que este aumento ponderal no es estable $\mathrm{y}$ sobre todo la inmunidad disminuye enormemente. Semejantes fracasos se observan en mezclas pobres en $H$. de $C$. o albúminas.

Cualquiera alimentación artificial defectuosa provoca un trastorno nutritivo, trastorno de la "vitalidad" del niño, como lo llama Bessau y como consecuencia de estas alteraciones se presentan las predisposiciones a las infecciones, diarreas, edemas, etc.

Un buen alimento artificial debe estar basado en el principio fundamental que contenga todos los elementos en proporciones recíprocas a las necesidades nutritivas del organismo, tratando de asemejarlas a las que existen en la leche de mujer. Tras este fin han ido los diferentes alimentos artificiales hasta ahora conocidos. Ultimamente, Bessau y sus colaboradores crecn haber encontrado una composición ali.menticia que lleva su nombre. que se asemeja a la leche materna y su cualidad principal consiste en poder cultivar an forma artificial la flora intestinal del bacilo bifidus, como se observa en la alimentación natural.

El alimento Bessau sería una leche al tercio, que tiene cocimiento de hígado o extracto hepático, además lactosa, cobre, glicerina, fierro y todas las principales vitaminas; para favorecer el desarrollo de la flora del bacilus bifidus se le agrega cistina en cierta proporción. Se recomienda para la alimentación normal. No tengo datos de cuál haya sido el resultado práctico de este alimento, llama sí la atención la complejidad de sus componentes y preparación.

En el alimento a base de leche de vaca debe tratarse de asemejar las proporciones de sus tres componentes principales, a la leche de mujer, o sea. $1 \%$ de albúmina, $3.5 \%$ de grasa y $7 \%$ de hidratos de carbono, sobre todo la proporción entri 
las grasas y los hidratos de carbono $(1: 2)$, dadas las relaciones que existen entre estos dos elementos que son antagonicas en el metabolismo orgánico. Sabemos que la grasa es de oxidación lenta, retrasa el metabolismo, en cambio, los hidratos de carbono, de oxidación rápida. lo acelera: la grasa inhibe la retención de agua, to que en cambio, es favorecida por los hidratos de carbono: la grasa da un aumento de peso estrble, el de los hidratos de carbono es inestable: la grasa aumenta la inmunidad y resistencia, no así los hidratos de carbono que aún la disminuyen cuando son dados en exceso. Estos antagonismos se compensan, procurando que hava proporción en las cantidades de hidratos de carbono y grasas como en la leche de mujer.

Las proporciones de albúmina no tienen la misma importancia que la de los otros elementos que acabamos de indicar en la alimentación del niño sano.

Fuera de la proporción de sus componentes en la alimentación artificial. hay que considerar la absorción intestinal de éstos, pues mientras más rápida sea, mayor garantía tendrá el niño, tanto por su mejor aprovechamiento en beneficio de su desarrollo general, como la repercusión que podría tener en la flora microbiana intestinal y por ende, en el mismo estado de salud.

La flora microbiand que se desarrolla en el intestino dapende de la clase de alimento y para ello debe usarse mezclas cuya absorción se haga en el intestino delgado. para que la acción de la flora microbiana quede limitada al intestino grueso; en esta forma, el intestino tolera la flora microbiana de la alimentación artificial con la misma inocuidad que la flera bífida de la alimentación natural.

No nos extendemos en mayores consideraciones al respecto, pues nuestro objetivo es exponer el resultado práctico con la alimentación artificial en el primer trimestre de la vida. Y prácticamente, hemos tratado de adaptar la composición del alimento artificial a la de la leche de mujer en cuanto a su roletancia y hemos administrado las cantidades sufícientes para satisfacer los requerimientos nutritivos necesarios.

Considerando la leche de mujer como alimento lóxico e ideal en los primeros meses de la vida, solamente prescribimos el alimento artificial por ecasez de aquél. Estimamos que la opinión pusilánime de los que la rechazan, es exagerada colocando a los padres en un disparadero cuando hacen cuestíón de vida o muerte el tener que dar leche humana al lactante, cuya madre carece del alimento necesario. Es cierto, que 
muchos niños no se adaptan al alimento heterólogo, o son de constitución débil, diatésicos; en éstos forzosamente bay que darles alimento natural.

Descartado el factor del niño mismo, los tracasos con la alimentación artificial las menos veces se deben al alimento, en cambio, en la mayoría de los casos, son consecuencias de ertores de técnica alimenticia y como dice Finkelstein, "la insuficiente alimentación es la causa no sólo de la mayor parte de los casos que se desarrollan mal, sino también de la mayor patte de todos los fracasos definitivos de la alimentación artificial, que alcanzan grados incurables o que ya no pueden vencerse más que empleando leche de mujer".

La hipo-alimentación se debe al temor con que se alimentan estos niños tan pequeños, al uso de leche muy diluída, al tomar como guía de sa asimilación el aspecto de las deposiciones, su número y consistencia, sin tomar en cuenta que casi siempre las diarreas que suelen observarse no se deben al alimento, sino que se trata de niños constitucionales. hidrolábiles, etc., que posiblemente las presentarian con la leche de mujer, o son consecuencias de la inanición por hipo-alimentación. las que se corrigen aumentando la ración allimenticia. Interpretar estas diarreas como trastorno dispéptico es un error que se comete a menudo; deposiciones frecuentes cuando faltan otros síntomas, no deben considerarse para restringir un régimen alimenticio por temor a una dispepsia.

Es fundamental que el alímento artificial llene en absoluto las necesidades nutritivas del niño. las que podemos controlar por su desarrollo en total, por ilos cálculos calorimétricos, conociendo los valores energéticos de sus compnnentes, darle el número de comidas necesarias, las que están sujetas a variaciones, según la calidad y tipo de niño, pues numerosos niños exigen mayores requerimientos calóricos a los corrientes, para hacer un desarrollo satisfactorio, Por último, exige una observación constante y personal, sobre todo en los niños asilados.

En la Casa Nacional del Niño usamos el Babeurre al $5 \%$, que es Babeurre con $5 \%$ de azúcar corriente. Babeurre al $7 \%$ con $5 \%$ de azúcar y $2 \%$ de harina flor. El primero contiene $2.63 \%$ de albúmina, $1.01 \%$ de grasa y $8.5 \%$ de hidratos de carbono: 550 calorías por litro; el segundo, $2.83 \%$ de albúmina, $1.01 \%$ de grasa y $10.5 \%$ de hidratos de carbono: 630 calorías por litro. Fritura de mantequilla (C. K.) : $0.44 \%$ de albúmina, $5.04 \%$ de grasas y $8.43 \%$ de hidratos de carbono: 830 calorías por litro; su composi- 
ción en 1000 grs. es: 5 grs. de mantequilla, 5 grs. de azúcar, 5 grs. de harina flor y 100 grs. de agua. Leche $2 / 3$ al $8 \%$, tiene $2.3 \%$ de albúmina, $2.4 \%$ de grasa, $11 \%$ de hidratos de carbono: 770 calorías por litro. Leche total al $10 \%$, tiene $3.48 \%$ de albúmina, $3.41 \%$ de grasa, $13.98 \%$ de hidratos de carbono: 1,030 calorías por litro.

El requerimiento en vitaminas lo reforzamos dándoles jugo de frutas en proporción a la tolerancia individual; las vitaminas $A$ y $D$ en gotas de cualquiera de los preparados existentes y recientemente se está ensayando el golpe antirraquítico como medida preventiva en contra del raquitismo, el que es muy frecuente en los niños asilados, sobre todo en los prematuros, a pesar de las medidas dietéticas e higiénicas que se toman para evitarlo.

Prácticamente hemos observado que la fritura de mantequilla no sólo mejora la inmunidad en el lactante durarte su uso, sino que la mantiene posteriormente cuando. de acuerdo con su mayor edad, se le instituye otra clase de alimentos.

En el niño, en los primeros meses de la vida, como dice Pfaundler, "no es posible proceder esquemáticamente, sino adaptar en cada caso el alimento a las necesidades individuales de cada niño". Esta adaptación debe ser considerada, con mayor razón, en los niños asilados, como pasa en la Casa $\mathrm{Na}$ cional del Niño que, por razones que sería abvio puntualizar, requieren mayor cuidado que el atendido individualmente y por eso las mezclas de lerhe que usamos no sün las simples que habitualmente se prescriben, sino que se le adiciona grasa precozmente y conforme a los conceptos de Finkelstein, ma. yor cantidad de hidratos de carbono y si consideramos su valor energético en relación al peso, generalmente reciben 150 o más calorías por kílo de peso.

No presentamos la estadística del total de niños criados artificialmente en el primer trimestre de la vida, en la Casa Nacional del Niño; cada dia son mayores; en parte. porque cada vez se hace más difícil obtener leche de mujer y porque conociendo con la experiencia mejor su técnica, se hace más fácil su aplicación.

Hemos resumido algunas observaciones. unas de niños tecién ingresados y otras de niños ya egresados.

A. Ch.-OBSERVIACION N:? 9627.

Ingresa de 5 horas de edad, con $3,020 \mathrm{grs}$, de pieso. con $0.49 \mathrm{~m}$. de longitud, $0.49 \mathrm{~m}$. de cirunfenncia craneana y $0.23 \mathrm{~m}$, de cincunferencia torícica. Hijo de madre tuberculosa, 
A su ingneso, se le da leche bumena exclusiva. Al 5.9 aía de edad. Babeure al $5 \% .500$ grs. diario y $200 \mathrm{grs}$, de ledhe ihnmana. AI mes de edad, $5 \times 100$ Bab., $5 \%$ más 20 grs. de C. K. Al mes y medio $5 \times 150$ B. C. $K$. al $1 / 2$ : a los 2 meses 4 x 150 B. C. K, al $1 / 2$ y $1 \times 150$ L. al $2 / 3$ al $8 \%$, 530 calorías, $137.7 \mathrm{cal} . \mathrm{Kg}$. Actualmente tiene $2 \frac{1}{2}$ meses $\mathrm{y}$ con aliment:ción $2 \times 150$ L. al $2 / 3$ al $8 \%, 3 \times 150$ B. C. K. al $1 / 2,564$ calorias, 149 cal $\mathrm{x} \mathrm{Kg}$.

El primer mes aumentó 180 grs. de peso y 3 cmss. de longitad: el stguindo mes, $750 \mathrm{grt}$. de peso y $2 \frac{1}{2}$ oms. de longitud. Como enfermedad intécarrente, ana rinofaringitis. Reaccionss a twberculina negativas.

B. del R. S. OBSERVACION N. 9404, K. 11 .

îngresa de 1 mes 20 dias con $2,700 \mathrm{grs} .0 .52 \mathrm{~m}$. de longitud, $0.35 \mathrm{~m}$. de circunférencia craneana y $0.32 \mathrm{~m}$. de circumferencia torácica. Madre: insuficiencia candiaca.

Alimentado exclusivamente por la madre.

Diagnóstito de ingreso: distrofia por hipoalimentación.

Se le instituye como almento: liche bumana y Bab. $5 \%$. A los 2 meses, Bab. $5 \% \times 5$; a $\operatorname{los} 2 \frac{1}{2}$ meses, 5 x 150 B. C. K. $1 / 2.558$ cal. 132.8 cal. $x \mathrm{Kg}$. Los 10 primeros días aumenta $210 \mathrm{gcs}$. de peso; ell 2.9 mes, $960 \mathrm{grs}$. y 3 cms. de longitud: no presenta enfermedades incitentrentes.

M. R. C. - CBSERVACION N. 9509. K. 168.

Ingrasa de 13 dias de edzd, con $2,500 \mathrm{grs}$. de peso, $0.47 \mathrm{~m}$. de longitud: $0.33 \mathrm{~m}$. de sincunferencia crane: na; $0.32 \mathrm{~m}$. de circunfírencia toráxica. Madie fatlece a consecuencia del parto.

A su ingreso se 12 instituye Bab. $5 \%$; a los 2 meses de edad. 6 x 100 . Bab. $5 \%$ más 30 C. K. $(549$ calorias. 152.5 caloxías por kilo de piso). A Ics 2 meses 10 dias: $5 \times 150$ B. C. K. $1 / 2 \quad 566$ calorias. 154 cal. $x$ kito de peso) A los 2 meses 20 dis: $2 \times 150$ L. $2 / 3$ al $8 \%, 3 \times 150$ B. C. K. $1 / 2$ (565 cal. 148 cal. $x$ kilo de peso). A los 3 meses peja 4 kilos: el primer mes crece $3 \mathrm{cms}$; ej $20^{\circ}$ nis 2 cms.

Como enfermødades intercurnentes prézenta rinitis serosa, piara y rinofaringitis.

M. F. - OBSERVACION N. 968 ?. K. 137.

Ingresa de 27 dias de edad, con 2,730 grs. de peso, $0.49 \mathrm{~m}$. de calla, $0.34 \mathrm{~m}$. de crounferencia cranasa y $0.30 \mathrm{~m}$. de cirzunferencia totácica.

Diagnóstico: Gemelo.

A su ingreso se le da $8 \times 60$ Bab. $5 \%$; 3 días deapués. $6 \times 100$ 5ab. $7 \%$ : al mes 20 dias de edad: $5 \times 100$ Bab. $5 \%$ más 20 C. K.; a los 2 mesis se aumenta el C. K. a 40 grs. (441 cal. 120 cal. $x$ kilo de pero), Det $10^{\circ}$ al $2 .{ }^{\circ}$ mes aumerta 880 gts.

Como enfermedid intercursente, rinofaringitis. 
P. G. V. - OB6ERVACION N.4 9387. K. 132.

Ingresa al mes de edad con $240 \mathrm{grs}$. de peso, $54.5 \mathrm{cms}$. de talla. 37.5 cms. de circunferencia craneana y 37.5 cms. de citounferencia torínica. Abandonado por la madre.

Es alimentado por su madrs basta los 3 diaj antes de sa ingreso, que le da lecbe de vaca diluida en agua. Przienca deposiciones abundantro y murosas. Se le insticuye $6 \times 120$ Bab. $5 \%$; 5 dias despurs, Bab. 7\%, Al mes 20 dias de edad: 5 x 120 B :b. $7 \%$ mis 20 C. K.; a los $2 \frac{1}{2}$ me.es: 5 x 150 B. C. K. al $1 / 2$ (724 calorías. 131.8 cal. $x$ kio dz peso). A los 3 meses: 5.630 grs. if se le tha 2 mamajaras de $160 \mathrm{~L}$. 'J, $10 \%$.

En 2 meses admenta 1.390 grs. y 6 cms. de tatla.

Como enfermedad inicrurrente, tinofaringitis.

Actualmente tiene 8 meses $y$ pose 8.550 grs.

S. A. D. - OBSERVACION N.P 9156, K. 19.

Ingresa de 9 días de edad. con 2.880 gts. de peso. 50.5 cms. de talla. $35 \mathrm{cms}$. dn circutfetencia craneana y 34 cms. de circanferencia torácica. Madre fallece de septicemia puemeral.

Se le institcye $6 \times 100 \mathrm{Bab} .5 \%$. A los 2 meses se agrega $30 \mathrm{C}$. K. $\times 6$. A los 3 meses: $3 \times 150$ B C. K. $1 / 2,2 \times 150 \mathrm{~J}: 2 / 3.8 \%$ A estiz edad pesa $4,770 \mathrm{grs}$.

No presenta enfermedades intersarente:

Abandona el estabotecimiento al año y 1 mas con $10.100 \mathrm{gTs}$ y $75 \mathrm{cms}$. de talla.

V. M. B. - OBSERVACION N.? $9104, \mathrm{~K}, 149$.

Ingresa de 1 mes 20 dias con 4.710 grs. he puso, 56.5 cms. de tatla. 28.5 cms." de circunferencia craneana y $39.5 \mathrm{cms}$. de circunferencia torácica.

Durante los 6 primeros dias se le da $5 \times 160$ Bab. $7 \%$ derpnés $5 \times 100$ B. C. K. $1 / 2$; a los 2 meses 5 días: $5 \times 180 \mathrm{~L} .2 / 3.8 \%$. A los mesas pera 5,400 grs. con $57.5 \mathrm{cms}$. de talla.

Abantiona el establecimiento, adoptado. 1 l $\% 9$ meses con $9600 \mathrm{gra}$, 65 cons. de talla.

J. A. P. - OBSERVACION N." 9474, K.

Ingresa de 2 dias de edad, con $3.300 \mathrm{grs}$. de peso, $52.5 \mathrm{cms}$ de talla, $36 \mathrm{oms}$, de cinounfetencia craniana y $32 \mathrm{cms}$. de circunferencia tord́cica.

Hijo de madze tuberculosa. separado al nacer.

Desde su ingreso se le da leothe hamana $y$ Bab. $5 \%$ ha:ta la edad de 2 aneses, que se le da: $3 \times 150,12 / 3,8 \%$ y $2 \times 150$ B. C. K. $1 / 2 ; 15$ días después la lecbe de vaca se da al $8 \%$ y a los 2 meses prea 4.910 grs, y 56 cms. de talla.

Permaneció en el establecimiento basta la edad de 4 meses y 7 días. Desando 5.670 grs y $63.5 \mathrm{cms}$. de talla a su tetiro, a pesar de hober presentado 
varias infecciones intercurrentes: difteria faringen $\gamma$ nasal, rinofaringitis $y$ otitis media.

L. A. - OBSERVACION N:\% 8650 .

Ingresa de 1 dia con 3.280 grs. de peso, 50 cms. de taitla, 34 cms. de circunferencia craneana y $35 \mathrm{cms}$, de circunferensia toraliva. Hijo de madre tuberculosa serarado al nacer.

Hasta los 20 días. lecbz humana con Bab. $5 \%$; is esa edad 6 x 100 Bab. $5 \%:$ al mes agregan 30 grs. de C. K. $x$ 6; al mes 20 dia: $6 \times 120$ B. C. K. $1 / 2$ : a $10 ; 2$ meses: $3 \times$ J $20 \mathrm{~L}, 2 / 3,8 \%$ y $3 \times 120$ B.C. K. $1 / 2$ : a los $21 / 3$ meses: $6 \times 120$ L. 2/3.8\%. A los 3 meses pesa 4,825 grs. con 59 cms. de talla.

Abandona of establecimicnto, envirdo a colocación familiar, a los 11 meses 8 dias. con $30,100 \mathrm{grs}, y 72 \mathrm{cms}$. 1 : tallia

\section{M. - OBSERVACION N. 8759 ,}

lngnesa de 13 dias, con $2,190 \mathrm{gra}, 46 \mathrm{cms}$, de talla. $34 \mathrm{cms}$, de ciccunferencia craneana y 34.5 cons. de citcunferenitiz torácica.

Ptematuro, Hijo de madrc tuberculoj 4 dias en contacto con su madre.

Drode su ingreso bata $1 \frac{1}{2}$ mes se le da lithe frumana y Bzb. $5 \%$; a esa edad 6 x 120 Bab. $5 \%$ más 30 C. K.; poco despuis el Báoaurra sa da al $7 \%$ : a 10 2 $2 \frac{1}{2}$ mes.s: $5 \times 120$ B. C. K. $1 / 2$.

A los 3 meses pesa 4,000 gr.s.; 53 cms. de talla.

Permanece en el es:ablecimiento basta los 6 meses con peso de $5,850 \mathrm{grs}$. y 63 wins. do talla. Adoptado.

F F. - OBSERVACION N.P 9139. K. 90.

Ingresa de 8 dias de edara, con $3,060 \mathrm{grs}$. de peso, 51 ims. de talla, 34 cmas. de circanferencia craneana y $33 \mathrm{cms}$. idz circunferencin torácica.

Hijo de madre tuberculosa siparado al notiet.

Hasta el mes de adad toma leobe humana y Bab. $5 \%$; al mes: 5 x 120 B $\approx b$. $5 \%$; al me; y medio se ingrega 30 grs. de C. K. a cada mamadera; a los $2 \frac{1}{2}$ meses: $5 \times 140$ Bab. $5 \%$ C. K. 1/2. A los 3 meses pesa $5,280 \mathrm{grs}$. con takla de 54 cms.

A los 10 meses abandona ostablecimiento con 9.300 grs. de peso y 72 cms. de talla.

\section{G. P. - OBSERVACION N.: 8652 .}

Ingresa de 15 días de edad con 3,400 grs. de peso; $52 \mathrm{~cm}$. de talla: 36 cms. de circunferencia craneant y 34 cms. de citicunferencia torákica. Abandonato.

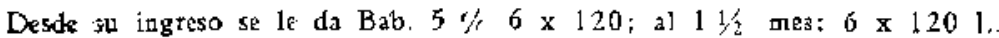
$2 / 3,8 \%$. A los 3 meses, pesa $5.600 \mathrm{grs}$ y 58 coms. de talla.

A los $61 / 2$ meses es adoptado. Paca 7.525 gr.. v $67 \mathrm{~cm}$. A: calla. 
R. del C. - OBSERVACION N. ${ }^{\circ} 8679 . \mathrm{K} .154$.

Ingresa de 1 mes 8 dias con $2,430 \mathrm{grs}$ de peso, $51.5 \mathrm{cms}$. de ta!1a, 36 cms. de ciccunferantia craneana y $35 \mathrm{cms}$. de citcanferencia torácica.

Hijo de madre tubenculoja. en contacto con ella durante 23 dias.

Los 10 primeros dias de su ingreso toma $6 \times 120 \mathrm{Bab} .7 \%$; después $6 \times 120 \mathrm{~L} .2 / 3$, $8 \%$. A los 3 meses pasa 4,675 grs. y 59 :ms, de talla.

Abandona el estableximiento a los 10 treses 22 dias con 9.300 grs. di peso y 71 cms. de talla.

M. A. V. - OBSER VACION N. 9493.

Ingresa de 1 día de eddat con 2.700 grs. de peso y 47.5 cms. de talta. Diagnóstico de ingreso: iRecién nacido l(abandonado).

Deste su ingreso se la dis lethe humana y Babeure $5 \%$. Durante el tercer mes se haces agregados de C, K. hasta llgar a B. C. K. 依.

El primet mes progresa 460 grs. de peso $\gamma 2 \frac{1}{1}$ ans. de talla: durartz fl segrando mes, 665 grs. y 4 ims. de talja y durance el tétcero, 500 grt. y 1 $\mathrm{cm}$. de talld. No presenta enfermedides intercurrentes.

\section{E. B. - OBSERVACION N.F 9447.}

Ingresa a los $14 \mathrm{~d}^{\prime} \mathrm{as} \mathrm{de}$ dad con 3,000 grs. de peso, $49 \mathrm{cms}$. dz ralla.

Diagnóstico de ingreso: Primigesto (forceps). Madte tabercslosa, en contacto con ella 48 horas.

Dasde su ingreso se le da leche hnmanı durante 19 dias, después Bab. $7 \%$. Durante el segundo mas. Bab. $10 \%$. A eita edad presenta una rinofaringitis y trastorno nurritivo agudo, que so trata con una dieta hidrita, crems de atroz, Bab. y lethe humana. Durante el tzreer mes se alimenta con Bab. $5 \%$. con agragidos de C. K. y finalmente B. C. K. $1 / 2$.

En el primer mes progresa 660 grs. de peso y $2 \mathrm{cms}$. de talla, en al sir gundo, 0 gr. y 2 cms. de talla $y$ en el tercero, 775 grs. y 1 cm, de talla.

\section{F. F. - OBSERVACION N." 9328.}

Ingresa de 14 idías de edad con 4.150 grs de peso y 53 cms, de talla.

Se instituye aljinentación con lecthe humina y 7 dias daspués Bab. 5 . durante el segundo mes se agrega C. K., pasando progresivamentr a B. C. K. l\%. En el tercer tmes se pasa a L. 2/3. $8 \%$, después de leche humana ty S. M. K.; a esta alad presenta di-pepsia y rinofaringitis. débiendo rarurtirse a una dieta bidrica, realimentación con suero de Bab. y crema de arroz. Como enfermcdades intercurrentes presenta, además, rinitis y otitis.

Durante el primer mes progresa $700 \mathrm{gts}$, de peso y $2 \frac{1}{2}$ cros. de longitud; en el segundo mes, 350 grs. de peso y 2 cmus. de longitad y en el tercero. 500 grs. de peso $F 1 \frac{1}{2}$ cms. de longitud.

J. de :a C. I. - OBSERVACION N. 9.623.

Ingresa a los 19 días de entad con $3,580 \mathrm{grs}$, de peso y 53 cms, de talla. 
Se institnye alimentaxión con Babente al $5 \%$ desde su ingreso: al mes de edad se le da Bab. $7 \%$; al mes y medio: Bab. $10 \%$. En el segundo mzs: 2 mamaderas da L. $2 / 3$. $8 \%$ y 3 inamaderas de B. C. K. $1 / 2$. En el tercer. mes: 5 mismaderas de L. $2 / 3.8 \%$.

En el primer mes progresa 870 grs. de peso $y 1 \mathrm{~cm}$. de longitud; $\mathrm{en}$ el segundo. 350 grs. de peso y $2 \frac{1}{2} \mathrm{cms}$, de longitud y en el tercero, $400 \mathrm{grs}$. de peso.

No prejunta enfermedades intercutrentes.

E. de a C. I. - OBSERVACION N. 9466.

Ingresa a la edad de 1 día con 3,560 grs. de peso y 52 cms. de talla.

Los primeros 20 d"as deqpués de su ungrese alimenta con lethe bumana y Bab. $5 \%$; después con Bub. $7 \%$. En el segundo mis con Bab. $7 \%$ exclusivo y en el tercer mes, agrogado dz C. K. 30 grs. y laego 60 grs.

Progresa 240 grs. y 2 oms. e] primer mes 500 grs. y 3 cms. el segundo y 400 grs. iy 1 cm. al terectro.

Como enfermedades intercutrentes precents, rinitis aguda serosa, rinofdringitis, otitis a repeticion, 